\title{
BOUNDEDNESS OF VALUE REGIONS AND CONVERGENCE OF CONTINUED FRACTIONS
}

\author{
F. A. ROACH
}

\begin{abstract}
If the elements of a continued fraction are restricted to lie within some region $E$ of the complex plane, it is quite of ten possible to determine, with very little difficulty, where the approximants of the continued fraction lie. Generally, it is more difficult to determine whether every continued fraction with elements from this set $E$ is convergent. In this paper, we give some results which, in certain cases, reduce the question of convergence to the question of whether the set of approximants is bounded.
\end{abstract}

1. We will be concerned with two forms of the continued fraction

$$
\frac{a_{1}}{b_{1}}+\frac{a_{2}}{b_{2}}+\frac{a_{3}}{b_{3}}+\cdots
$$

where each $a_{p}$ and $b_{p}$ is a complex number. We say that (1.1) is of type (A) provided that each $b_{p}$ has value 1 and that it is of type (B) provided that each $a_{p}$ has value 1. For certain computations, we will use the fundamental recurrence relations:

$$
\begin{aligned}
A_{-1} & =B_{0}=1, \quad B_{-1}=A_{0}=0 \\
A_{p} & =b_{p} A_{p-1}+a_{p} A_{p-2} ; \\
B_{p} & =b_{p} B_{p-1}+a_{p} B_{p-2}, \quad p=1,2,3, \ldots .
\end{aligned}
$$

It follows that for $p=1,2,3, \ldots$,

$$
\frac{A_{p}}{B_{p}}=\frac{a_{1}}{b_{1}}+\frac{a_{2}}{b_{2}}+\cdots+\frac{a_{p}}{b_{p}}
$$

and

$$
A_{p-1} B_{p}-A_{p} B_{p-1}=(-1)^{p} a_{1} a_{2} \cdots a_{p} .
$$

As is customary, we say that (1.1) converges provided that there are not infinitely many values of $p$ such that $B_{p}=0$ and the sequence $\left\{A_{p} / B_{p}\right\}$ converges to a finite limit.

If $E$ is a set of complex numbers, we use the symbol $V_{A}(E)$ to denote the set of all complex numbers of the form

$$
\frac{a_{1}}{1}+\frac{a_{2}}{1}+\cdots+\frac{a_{n}}{1}
$$

Presented to the Society, March 20, 1975; received by the editors June 30, 1975.

AMS (MOS) subject classifications (1970). Primary 30A22, 40A15.

Key words and phrases. Continued fractions, convergence regions for continued fractions, value regions for continued fractions.

() American Mathematical Society 1977 
where each one of $a_{1}, a_{2}, \ldots, a_{n}$ lies in $E$; the symbol $V_{B}(E)$ denotes the set of all complex numbers of the form

$$
\frac{1}{b_{1}}+\frac{1}{b_{2}}+\cdots+\frac{1}{b_{n}}
$$

where each one of $b_{1}, b_{2}, \ldots, b_{n}$ lies in $E$. We say that the set $E$ of complex numbers is a type (A) convergence set provided that if $a_{p}$ lies in $E$ and $b_{p}=1$, $p=1,2,3, \ldots,(1.1)$ converges; we say that it is a type (B) convergence set provided that if $b_{p}$ lies in $E$ and $a_{p}=1, p=1,2,3, \ldots,(1.1)$ converges. If $M$ is a set of complex numbers, the symbols $M+M$ and $M \cdot M$ denote, respectively, the set of all sums $x+y$ and all products $x y$ with $x$ in $M$ and $y$ in $M$.

2. In this section, we will prove the following two theorems.

TheOREM A. Suppose that $E$ is a set of complex numbers such that -1 is not a limit point of $V_{A}(E)+V_{A}(E)$. Then $E$ is a type (A) convergence set if and only if $V_{A}(E)$ is bounded.

TheOREM B. Suppose that $E$ is a set of complex numbers such that -1 is not a limit point of $V_{B}(E) \cdot V_{B}(E)$. Then $E$ is a type (B) convergence set if and only if $V_{B}(E)$ is bounded.

As would be expected, the proofs of these theorems are similar. We will give a proof of Theorem $\mathrm{A}$ and indicate, at certain points, the modifications which can be made to obtain a proof of Theorem B.

Suppose that $E$ is a set of complex numbers such that -1 is not a limit point of $V_{A}(E)+V_{A}(E)$ and $V_{A}(E)$ is bounded. Under this hypothesis, no $B_{p}$ has value 0 . To see that this is true, suppose that for some $p, B_{p}=0$ and let $k$ denote the least such value of $p$. From (1.2), $B_{1}=1$ and $B_{2}=1+a_{2}$. If $B_{2}$ were 0 , then -1 would be in $E$ and hence, $-1 /(1+(-1 / 1))=\infty$ would be in $V_{A}(E)$. Therefore, $k>2$. But $B_{k}=B_{k-1}+a_{k} B_{k-2}=0$ so that $a_{k}=$ $-B_{k-1} / B_{k-2}$. By (1.2),

$$
a_{k}=-\left(1+\frac{a_{k-1}}{B_{k-2} / B_{k-3}}\right) .
$$

Continuing in this manner, we find that

$$
a_{k}=-\left(1+\frac{a_{k-1}}{1}+\frac{a_{k-2}}{1}+\cdots+\frac{a_{1}}{1}\right) \text {. }
$$

By a simple calculation, we see that if $a$ is any complex number distinct from 0 ,

$$
\frac{a}{1}+\frac{a_{1}}{1}+\frac{a_{2}}{1}+\cdots+\frac{a_{k-1}}{1}+\frac{a_{k}}{1}=\infty .
$$

Therefore, no $B_{p}$ has value 0 .

With regard to Theorem $\mathrm{B}$, we see that 0 is not in $E$ and since $A_{2} / B_{2}=$ $b_{2} /\left(b_{1} b_{2}+1\right), B_{2} \neq 0$. From the equation $B_{k}=b_{k} B_{k-1}+B_{k-2}=0$, it follows that 


$$
b_{k}=\frac{1}{-b_{k-1}}+\frac{1}{-b_{k-2}}+\cdots+\frac{1}{-b_{1}}
$$

and hence,

$$
\frac{1}{b_{1}}+\frac{1}{b_{2}}+\cdots+\frac{1}{b_{k-1}}+\frac{1}{b_{k}}=\infty \text {. }
$$

The number -1 does not belong to $V_{A}(E)+V_{A}(E)$, for if it did, then there would be a sequence $a_{1}, a_{2}, \ldots, a_{n}, a_{1}^{\prime}, a_{2}^{\prime}, \ldots, a_{m}^{\prime}$ from $E$ such that

$$
\frac{a_{1}}{1}+\frac{a_{2}}{1}+\cdots+\frac{a_{n}}{1}=-1-\left(\frac{a_{1}^{\prime}}{1}+\frac{a_{2}^{\prime}}{1}+\cdots+\frac{a_{m}^{\prime}}{1}\right) \text {. }
$$

Then for any nonzero $a$, we would have

$$
\frac{a}{1}+\frac{a_{m}^{\prime}}{1}+\cdots+\frac{a_{1}^{\prime}}{1}+\frac{a_{1}}{1}+\cdots+\frac{a_{n}}{1}=\infty .
$$

For Theorem B, if

$$
\left(\frac{1}{b_{1}}+\frac{1}{b_{2}}+\cdots+\frac{1}{b_{n}}\right) \cdot\left(\frac{1}{b_{1}^{\prime}}+\frac{1}{b_{2}^{\prime}}+\cdots+\frac{1}{b_{m}^{\prime}}\right)=-1,
$$

then

$$
\frac{1}{b_{m}^{\prime}}+\frac{1}{b_{m-1}^{\prime}}+\cdots+\frac{1}{b_{1}^{\prime}}+\frac{1}{b_{1}}+\cdots+\frac{1}{b_{n}}=\infty
$$

Also, under the hypothesis above, there exist positive numbers $a$ and $b$ such that if each of $x$ and $y$ is in $V_{A}(E), a \leqslant|1+x+y| \leqslant b$. Therefore, there is a positive number $M$ such that if each $x, y, x^{\prime}$, and $y^{\prime}$ is in $V_{A}(E)$, then

$$
M|1+x+y| \leqslant\left|1+x^{\prime}+y^{\prime}\right|
$$

Suppose that each one of $a_{1}, a_{2}, a_{3}, \ldots$ lies in $E$. For $p=1,2,3, \ldots$, let $t_{p}(z)=a_{p} /(1+z)$ and $s_{p}(z)=t_{1} t_{2} \cdots t_{p}(z)$ so that $s_{p}(0)=A_{p} / B_{p}$. Let $n_{1}$, $n_{2}, n_{3}, \ldots$ and $k_{1}, k_{2}, k_{3}, \ldots$ be increasing sequences of positive integers such that $n_{p}+2<k_{p}<n_{p+1}, p=1,2,3 \ldots$ Then, for $p=1,2,3, \ldots$, we have

$$
\begin{aligned}
M \cdot \mid(1+ & \left.\frac{a_{n_{p}+1}}{1}+\cdots+\frac{a_{1}}{1}\right)+\left(\frac{a_{n_{p}+2}}{1}+\cdots+\frac{a_{n_{p+1}}}{1}\right) \mid \\
& \leqslant\left|\left(1+\frac{a_{n_{p}+1}}{1}+\cdots+\frac{a_{1}}{1}\right)+\left(\frac{a_{n_{p}+2}}{1}+\cdots+\frac{a_{k_{p}}}{1}\right)\right|
\end{aligned}
$$

and, referring to (1.2),

$$
\frac{M}{\left|B_{n_{p}+1} / B_{n_{p}}+t_{n_{p}+2} \cdots t_{k_{p}}(0)\right|} \leqslant \frac{1}{\left|B_{n_{p}+1} / B_{n_{p}}+t_{n_{p}+2} \cdots t_{n_{p+1}}(0)\right|} .
$$

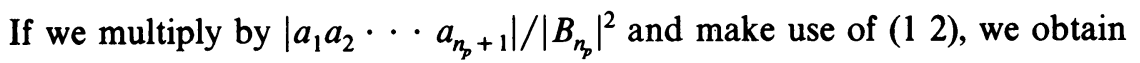

$$
\frac{M\left|a_{1} a_{2} \cdots a_{n_{p}}\right||x|}{\left|B_{n_{p}}\right|\left|B_{n_{p}}+x B_{n_{p}-1}\right|} \leqslant \frac{\left|a_{1} a_{2} \cdots a_{n_{p}}\right||y|}{\left|B_{n_{p}}\right|\left|B_{n_{p}}+y B_{n_{p}-1}\right|}
$$

where 


$$
x=\frac{a_{n_{p}+1}}{1}+\cdots+\frac{a_{k_{p}}}{1} \text { and } y=\frac{a_{n_{p}+1}}{1}+\cdots+\frac{a_{n_{p+1}}}{1}
$$

However, this is the same as

$$
M\left|s_{n_{p}}(0)-s_{k_{p}}(0)\right| \leqslant\left|s_{n_{p}}(0)-s_{n_{p+1}}(0)\right| .
$$

Since $V_{A}(E)$ is bounded, some subsequence of $\left\{s_{p}(0)\right\}_{p=1}^{\infty}$ is convergent and, with (2.2), we see that no two subsequences converge to different limits. Therefore, $E$ is a type (A) convergence set.

The proof for Theorem B is quite similar. Rather than (2.1) we have

$$
M|1+x y| \leqslant\left|1+x^{\prime} y^{\prime}\right| \text {. }
$$

If we now suppose that for $p=1,2,3, \ldots, b_{p}$ lies in $E, t_{p}(z)=1 /\left(b_{p}+z\right)$, and $s_{p}(. z)=t, t_{2} \cdots t_{p}(z)$, we have

$$
\frac{M}{\left|1+t_{n_{p}+1} \cdots t_{k_{p}}(0) B_{n_{p}} / B_{n_{p+1}}\right|} \leqslant \frac{1}{\left|1+t_{n_{p}+1} \cdots t_{n_{p+1}}(0) B_{n_{p}} / B_{n_{p}-1}\right|} .
$$

Multiplying by $\left|B_{n_{p}}\right|\left|B_{n_{p}+1}\right|$ and making use of (1.2), we have, as before, (2.2).

Now suppose that $E$ is a set of complex numbers such that -1 is not a limit point of $V_{A}(E)+V_{A}(E)$ and that $E$ is a type (A) convergence set. If $V_{A}(E)$ were not bounded, there would exist a sequence $a_{1}^{1}, a_{1}^{2}, a_{2}^{2}, a_{1}^{3}, a_{2}^{3}$, $a_{3}^{3}, \ldots$, with each term in $E$ such that

$$
\frac{a_{1}^{1}}{1}, \frac{a_{1}^{2}}{1}+\frac{a_{2}^{2}}{1}, \frac{a_{1}^{3}}{1}+\frac{a_{2}^{3}}{1}+\frac{a_{3}^{3}}{1}, \ldots
$$

converges to $\infty$. As is well known, in order that $E$ be a type (A) convergence set it is necessary that it be bounded so that the sequence $a_{1}^{1}, a_{1}^{2}, a_{1}^{3}, \ldots$ does not increase without bound and, hence, the sequence of reciprocals of (2.4) must converge to 0 . From this it follows that the sequence

$$
\frac{a_{2}^{2}}{1}, \frac{a_{2}^{3}}{1}+\frac{a_{3}^{3}}{1}, \frac{a_{2}^{4}}{1}+\frac{a_{3}^{4}}{1}+\frac{a_{4}^{4}}{1}, \ldots
$$

converges to -1 . For any nonzero complex number $a$, the sequence obtained by applying the transformation $a /(1+z)$ to each term of $(2.4)$ converges to 0 . If such a sequence is added term by term to the sequence above, we see that -1 is a limit point of $V_{A}(E)+V_{A}(E)$.

In the case of Theorem $\mathrm{B}, E$ must be bounded away from 0 and from the assumption that

$$
\frac{1}{b_{1}^{1}}, \frac{1}{b_{1}^{2}}+\frac{1}{b_{2}^{2}}, \frac{1}{b_{1}^{3}}+\frac{1}{b_{2}^{3}}+\frac{1}{b_{3}^{3}}, \ldots
$$

converges to $\infty$, it follows that

$$
\frac{1}{b_{1}^{2}} \cdot \frac{1}{b_{2}^{2}}, \frac{1}{b_{1}^{3}} \cdot\left(\frac{1}{b_{2}^{3}}+\frac{1}{b_{3}^{3}}\right), \frac{1}{b_{1}^{4}} \cdot\left(\frac{1}{b_{2}^{4}}+\frac{1}{b_{3}^{4}}+\frac{1}{b_{4}^{4}}\right), \ldots
$$

converges to -1 . 
3. For real numbers, we have the following stronger results.

THEOREM $\mathrm{A}^{\prime}$. Suppose that $E$ is a set of real numbers. Then $E$ is a type (A) convergence set if and only if $V_{A}(E)$ is bounded.

THEOREM B'. Suppose that $E$ is a set of real numbers. Then $E$ is a type (B) convergence set if and only if $V_{B}(E)$ is bounded.

Suppose that $a$ is a real number less than $-1 / 4$. Let $u$ denote $1 / 2+$ $i(-1-4 a)^{1 / 2} / 2$ and let $v$ denote $u^{2} /|u|^{2}$. If, for $p=1,2,3, \ldots, a_{p}=a$ and $b_{p}=1$, then

$$
\frac{A_{n}}{B_{n}}=-\frac{\bar{u}}{v+1 / \sum_{p=0}^{n-1} v^{p}}
$$

(see [4, p. 23]). The denominator of this expression can be written as $v+(1-v) /\left(1-v^{n}\right)$. If $v$ is a root of unity, then for some $n, v^{n}=1 / v$, in which case this denominator is 0 . If $v$ is not a root of unity, the sequence $\left\{v^{n}\right\}$ is dense in the unit circle and hence some subsequence of these denominators converges to 0 . From these considerations, we see that if $V_{A}(E)$ is bounded, $E$ does not contain a real number less than $-\frac{1}{4}$. Since $E$ must be a subset of $V_{A}(E)$, when $V_{A}(E)$ is bounded, so is $E$. However, it is well known that if $E$ is a bounded set of real numbers and no element of $E$ is less than $-\frac{1}{4}$, then $E$ is a type (A) convergence set (see, for example, [4, Theorem 14.2 , p. 58]). It is also easily shown that if each element of the bounded set $E$ is a real number not less than $-\frac{1}{4}$, then $V_{A}(E)$ is bounded. If $E$ contains an element less than $-\frac{1}{4}$ or is unbounded, it is not a type (A) convergence set. Thus, we see that Theorem $\mathrm{A}^{\prime}$ is true.

Suppose that $p$ and $q$ are numbers such that $-4 / p<q<0<p$. Then $1 / p q<-1 / 4$. Since

$$
\frac{1}{b_{1}}+\frac{1}{b_{2}}+\cdots+\frac{1}{b_{n}}=\frac{a_{1}}{1}+\frac{a_{2}}{1}+\cdots+\frac{a_{n}}{1}
$$

where $a_{1}=1 / b_{1}$ and $a_{p}=1 / b_{p-1} b_{p}, p=2,3,4, \ldots$, it is not possible for both $p$ and $q$ to belong to $E$ and $V_{A}(E)$ to be bounded. If $V_{B}(E)$ is bounded, then $E$ is bounded away from 0 . If there exists a positive number $p$ such that no number between $-4 / p$ and $p$ belongs to $E$ and each element of $E$ is real, then $E$ is a type (B) convergence set (see, for example, [3, Corollary 2.2]). It is also true that every such set $E$ is a type (B) convergence set with $V_{B}(E)$ bounded.

4. Let $P$ denote the set of all complex numbers $z$ such that $|z|-\operatorname{Re}(z) \leqslant \frac{1}{2}$. By the Parabola Theorem of Scott and Wall [1] (Theorem 14.2 of [4]), if $E$ is a bounded subset of $P$, then $E$ is a type (A) convergence set. While Theorem A does not yield this result, it can be used to obtain a somewhat weaker result. Namely, if $E$ is a bounded subset of $P$ which is bounded away from the parabola, then it is a type (A) convergence set. It is quite easy to show that for such a set $E, V_{A}(E)$ is a bounded subset of the half-plane $\operatorname{Re}(z) \leqslant-\frac{1}{2}$ and that it is bounded away from the line $\operatorname{Re}(z)=-\frac{1}{2}$. Similar results can be obtained for certain "rotated" parabolic discs considered by Thron [2]. 
However, his General Parabola Theorem of that paper yields sharper results. Let $c$ be a real number and let $C$ be the set of all complex numbers $z$ such that $|z-c| \leqslant\left(c^{2}+4\right)^{1 / 2}$. In this case $V_{B}(C)$ is the set of all complex numbers $z$ such that $\left|z+\frac{1}{2} c\right|<\frac{1}{2}\left(c^{2}+4\right)^{1 / 2}$. If $E$ is a subset of $C$ which is bounded away from the boundary of $C$ (or, even bounded away from the common part of the joundary of $C$ and the half-plane $\operatorname{Re}(z) \leqslant 0),-1$ is not a limit point of $V_{B}(E) \cdot V_{B}(E)$. Thus, by Theorem $\mathrm{B}$, such a set $E$ is a type (B) convergence set. However, $C$ itself is a type (B) convergence set by Corollary 2.2 of [2].

In view of these examples and the results of $\$ 3$, one might conjecture that the boundedness of the value region alone is necessary and sufficient for a set of complex numbers to be a convergence set. Unfortunately, we are unable to settle this question at this time.

\section{REFERENCES}

1. W. T. Scott and H. S. Wall, A convergence theorem for continued fractions, Trans. Amer. Math. Soc. 47 (1940), 155-172. MR 1, 217.

2. W. J. Thron, On parabolic convergence regions for continued fractions, Math. Z. 69 (1958), 173-182. MR 20 \#2562.

3., Twin convergence regions for continued fractions $b_{0}+K\left(1 / b_{n}\right)$. II, Amer. J. Math. 71 (1949), 112-120. MR 10, 292.

4. H. S. Wall, Analytic theory of continued fractions, Van Nostrand, Princeton, N. J., 1948. MR $10,32$.

Department of Mathematics, University of Houston, Houston, TeXas 77004 